International Journal of Pure and Applied Mathematics

Volume 87 No. 4 2013, 611-619

ISSN: 1311-8080 (printed version); ISSN: 1314-3395 (on-line version)

url: http://www.ijpam.eu

doi: http://dx.doi.org/10.12732/ijpam.v87i4.10

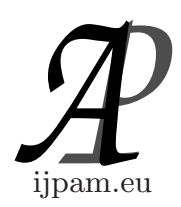

\title{
DIFFERENTIAL RECURRENCE RELATION OF GENERALIZED $K$-WRIGHT FUNCTION
}

\author{
Kuldeep Singh Gehlot ${ }^{1}$, Jyotindra C. Prajapati ${ }^{2}$, Bhailal P. Patel ${ }^{3}$ \\ ${ }^{1}$ Department of Mathematics \\ Government Bangur \\ P.G. College, Pali, 306401, Rajasthan, INDIA \\ ${ }^{2}$ Department of Mathematical Sciences \\ Charotar University of Science and Technology \\ Changa, Anand, 388421, Gujarat, INDIA \\ ${ }^{3}$ Department of Mathematical Sciences \\ N.V. Patel College of Pure and Applied Sciences \\ Vallabh Vidyanagar, Gujarat, INDIA
}

\begin{abstract}
The principal aim of this paper is to establish differential recurrence relation and Integral representation of generalized $K$-Wright function ${ }_{p} \Psi_{q}^{k}(z)$ introduced by Gehlot and Prajapati [2]. In the end some interesting special cases have also been discussed.
\end{abstract}

AMS Subject Classification: generalized $K$-Wright function, $K$-gamma function, Mittag-Leffler funtion

Key Words: 26A33, 33B15, 33C20, 33E12

\section{Introduction}

Generalized $K$-Gamma function $\Gamma_{k}(x)$ defined as (see Diaz and Pariguan [1])

$$
\Gamma_{k}(x)=\lim _{n \rightarrow \infty} \frac{n ! k^{n}(n k)^{\frac{x}{k}-1}}{(x)_{n, k}}, k>0, x \in \mathbb{C} \backslash k \mathbb{Z}^{-},
$$

where $(x)_{n, k}$ is the k-Pochhammer symbol and is given by

Received: June 25, 2013

(c) 2013 Academic Publications, Ltd. url: www.acadpubl.eu

${ }^{\S}$ Correspondence author 


$$
(x)_{n, k}=x(x+k)(x+2 k) \ldots \ldots \ldots(x+(n-1) k),
$$

where $x \in \mathbb{C}, k \in \mathbb{R}, n \in \mathbb{N}^{+}$. For $\operatorname{Re}(x)>0, \Gamma_{k}(x)$ is $K$-Gamma function defined as the integral

$$
\Gamma_{k}(x)=\int_{0}^{\infty} t^{x-1} e^{\frac{-t^{k}}{k}} d t
$$

It is easy to prove following results

$$
\begin{gathered}
\Gamma_{k}(x)=k^{\frac{x}{k}-1} \Gamma\left(\frac{x}{k}\right), \\
\Gamma_{k}(x+k)=x \Gamma_{k}(x), \\
(\gamma)_{r, k}=(k)^{r}\left(\frac{\gamma}{k}\right)_{r}, \\
(\gamma)_{r, k}=\frac{\Gamma_{k}(\gamma+r k)}{\Gamma_{k}(\gamma)}, \\
r k(x)_{r-1, k}=(x)_{r, k}-(x-k)_{r, k}, \\
(\delta)_{n+j, k}=(\delta)_{j, k}(\delta+j k)_{n, k} .
\end{gathered}
$$

\section{Generalized $K$-Wright Function}

Concept of the generalized $K$-Wright function introduced by Gehlot and Prajapati [2] as:

Definition. The generalized $K$-Wright function is defined as ${ }_{p} \Psi_{q}^{k}(z)$ for $k \in$ $\mathbb{R}^{+} ; z \in \mathbb{C}, a_{i}, b_{j} \in \mathbb{C}, \alpha_{i}, \beta_{j} \in \mathbb{R}\left(\alpha_{i}, \beta_{j} \neq 0 ; i=1,2, \ldots, p ; j=1,2, \ldots, q\right)$ and $\left(a_{i}+\alpha_{i} n\right),\left(b_{j}+\beta_{j} n\right) \in \mathbb{C} \backslash k \mathbb{Z}^{-}$,

$$
{ }_{p} \Psi_{q}^{k}(z)={ }_{p} \Psi_{q}^{k}\left[\begin{array}{c}
\left(a_{i}, \alpha_{i}\right)_{1, p} \\
\left(b_{j}, \beta_{j}\right)_{1, q}
\end{array} \mid z\right]=\sum_{n=0}^{\infty} \frac{\prod_{i=1}^{p} \Gamma_{k}\left(a_{i}+\alpha_{i} n\right) z^{n}}{\prod_{j=1}^{q} \Gamma_{k}\left(b_{j}+\beta_{i} n\right) n !} .
$$

For convergence, we use the following notations

$$
\Delta=\sum_{j=1}^{q}\left(\frac{\beta_{j}}{k}\right)-\sum_{i=1}^{p}\left(\frac{\alpha_{i}}{k}\right), \quad \delta=\prod_{i=1}^{p}\left|\frac{\alpha_{i}}{k}\right|^{\frac{-\alpha_{i}}{k}} \prod_{j=1}^{q}\left|\frac{\beta_{j}}{k}\right|^{\frac{\beta_{j}}{k}},
$$




$$
\mu=\sum_{j=1}^{q}\left(\frac{b_{j}}{k}\right)-\sum_{i=1}^{p}\left(\frac{a_{i}}{k}\right)+\frac{p-q}{2} .
$$

Theorem 2.1. For $k \in \mathbb{R}^{+} ; z \in \mathbb{C}, a_{i}, b_{j} \in \mathbb{C}, \alpha_{i}, \beta_{j} \in \mathbb{R}\left(\alpha_{i}, \beta_{j} \neq 0 ; i=\right.$ $1,2, \ldots, p ; j=1,2, \ldots, q)$ and $\left(a_{i}+\alpha_{i} n\right),\left(b_{j}+\beta_{j} n\right) \in \mathbb{C} \backslash k \mathbb{Z}^{-}$,

(a) If $\Delta>-1$ then series (10) is absolutely convergent for all $z \in \mathbb{C}$ and generalized $K$-Wright function ${ }_{p} \Psi_{q}^{k}(z)$ is an entire function of $z$.

(b) If $\Delta=-1$ then series (10) is absolutely convergent for all $|z|<\delta$ and of $|z|=\delta, \operatorname{Re}(\mu)>\frac{1}{2}$.

\section{Differential Recurrence Relation}

In this section we evaluate the recurrence relation of generalized $K$-Wright function, ${ }_{p} \Psi_{q}^{k}(z)$.

Theorem 3.1. For $k \in \mathbb{R}^{+} ; z \in \mathbb{C} ; a_{i}, b_{j}, \beta+s \in \mathbb{C} ; \operatorname{Re}(\beta+s)>0$ and $\alpha+\gamma, \alpha_{i}, \beta_{j} \in \mathbb{R}\left(\alpha_{i}, \beta_{j} \neq 0 ; i=1,2, \ldots, p ; j=1,2, \ldots, q\right)$, then

$$
\begin{aligned}
& { }_{p} \Psi_{q+1}^{k}\left[\begin{array}{c|c}
\left(a_{i}, \alpha_{i}\right)_{1, p} \\
\left(b_{j}, \beta_{j}\right)_{1, q},(\beta+s+k, \alpha+r)
\end{array} \mid z\right] \\
& -k_{p} \Psi_{q+1}^{k}\left[\begin{array}{c|c}
\left(a_{i}, \alpha_{i}\right)_{1, p} \\
\left(b_{j}, \beta_{j}\right)_{1, q},(\beta+s+2 k, \alpha+r)
\end{array} \mid z\right] \\
& =z^{2}(\alpha+\gamma)^{2} \ddot{\Psi}_{q+1}^{k}\left[\begin{array}{c|c}
\left(a_{i}, \alpha_{i}\right)_{1, p} \\
\left(b_{j}, \beta_{j}\right)_{1, q},(\beta+s+3 k, \alpha+r)
\end{array} \mid z\right] \\
& +z\left\{(\alpha+\gamma)^{2}+2(\alpha+\gamma)(\beta+s+k)\right\}_{p} \dot{\Psi}_{q+1}^{k}\left[\begin{array}{c}
\left(a_{i}, \alpha_{i}\right)_{1, p} \\
\left(b_{j}, \beta_{j}\right)_{1, q},(\beta+s+3 k, \alpha+r)
\end{array} \mid z\right] \\
& +(\beta+s)(\beta+s+2 k)_{p} \Psi_{q+1}^{k}\left[\left(b_{j}, \beta_{j}\right)_{1, q},(\beta+s+3 k, \alpha+r) \mid z\right] .
\end{aligned}
$$

Proof. From definition (10) and (5), we have

$$
\begin{aligned}
{ }_{p} \Psi_{q+1}^{k}\left[\begin{array}{c}
\left(a_{i}, \alpha_{i}\right)_{1, p} \\
\left(b_{j}, \beta_{j}\right)_{1, q},(\beta+s+k, \alpha+\gamma)
\end{array} \mid z\right] \\
=\sum_{n=0}^{\infty} \frac{\prod_{i=1}^{p} \Gamma_{k}\left(a_{i}+\alpha_{i} n\right)}{\prod_{j=1}^{q} \Gamma_{k}\left(b_{j}+\beta_{j} n\right)} \frac{z^{n}}{n !} \frac{1}{(\beta+s+(\alpha+\gamma) n) \Gamma_{k}(\beta+s+(\alpha+\gamma) n)}
\end{aligned}
$$


So

$$
\begin{aligned}
& p_{q+1}^{k}\left[\begin{array}{c}
\left(b_{j}, \beta_{j}\right)_{1, q},(\beta+s+2 k, \alpha+\gamma) \\
\left.\Psi_{i}\right)_{1, p}
\end{array}\right]=\sum_{n=0}^{\infty} \frac{\prod_{i=1}^{p} \Gamma_{k}\left(a_{i}+\alpha_{i} n\right)}{\prod_{j=1}^{q} \Gamma_{k}\left(b_{j}+\beta_{j} n\right)} \frac{z^{n}}{n !} \\
& \quad \times \frac{1}{(\beta+s+k+(\alpha+\gamma) n)(\beta+s+(\alpha+\gamma) n) \Gamma_{k}(\beta+s+(\alpha+\gamma) n)}
\end{aligned}
$$

this can be reduces to

$$
\begin{aligned}
& =\sum_{n=0}^{\infty} \frac{\prod_{i=1}^{p} \Gamma_{k}\left(a_{i}+\alpha_{i} n\right)}{\prod_{j=1}^{q} \Gamma_{k}\left(b_{j}+\beta_{j} n\right)} \frac{z^{n}}{n !} \frac{1}{\Gamma_{k}(\beta+s+(\alpha+\gamma) n)} \\
& \quad \times \frac{1}{k}\left\{\frac{1}{(\beta+s+(\alpha+\gamma) n)}-\frac{1}{(\beta+s+k+(\alpha+\gamma) n)}\right\} .
\end{aligned}
$$

Using (12), we can write

$$
\begin{aligned}
& S={ }_{p} \Psi_{q+1}^{k}\left[\begin{array}{c}
\left(a_{i}, \alpha_{i}\right)_{1, p} \\
\left(b_{j}, \beta_{j}\right)_{1, q},
\end{array}\right.(\beta+s+k, \alpha+\gamma) \mid z] \\
&-k_{p} \Psi_{q+1}^{k}\left[\begin{array}{c}
\left(a_{i}, \alpha_{i}\right)_{1, p} \\
\left(b_{j}, \beta_{j}\right)_{1, q},(\beta+s+2 k, \alpha+\gamma)
\end{array}\right] z
\end{aligned}
$$

where

$$
S=\sum_{n=0}^{\infty} \frac{\prod_{i=1}^{p} \Gamma_{k}\left(a_{i}+\alpha_{i} n\right)}{\prod_{j=1}^{q} \Gamma_{k}\left(b_{j}+\beta_{j} n\right)} \frac{z^{n}}{n !} \frac{1}{(\beta+s+k+(\alpha+\gamma) n) \Gamma_{k}(\beta+s+(\alpha+\gamma) n)}
$$

Now, applying a simple identity $\frac{1}{u}=\frac{k}{u(u+k)}+\frac{1}{u+k}$; for $u=\beta+s+k+n(\alpha+p)$ to $(12)$, we obtain

$$
\begin{aligned}
& S=\sum_{n=0}^{\infty} \frac{\prod_{i=1}^{p} \Gamma_{k}\left(a_{i}+\alpha_{i} n\right)}{\prod_{j=1}^{q} \Gamma_{k}\left(b_{j}+\beta_{j} n\right)} \frac{z^{n}}{n !} \frac{1}{\Gamma_{k}(\beta+s+(\alpha+\gamma) n)} \\
& \times\left\{\frac{k}{(\beta+s+k+(\alpha+\gamma) n)((\beta+s+2 k+(\alpha+\gamma) n))}+\frac{1}{(\beta+s+2 k+(\alpha+\gamma) n)}\right\} .
\end{aligned}
$$


This leads to

$$
\begin{aligned}
S & =\sum_{n=0}^{\infty} \frac{\prod_{i=1}^{p} \Gamma_{k}\left(a_{i}+\alpha_{i} n\right)}{\prod_{j=1}^{q} \Gamma_{k}\left(b_{j}+\beta_{j} n\right)} \frac{z^{n}}{n !} \times \\
& \left\{\frac{k(\beta+s+(\alpha+\gamma) n)}{(\beta+s+2 k+(\alpha+\gamma) n)(\beta+s+k+(\alpha+\gamma) n)(\beta+s+(\alpha+\gamma) n) \Gamma_{k}((\beta+s+(\alpha+\gamma) n))}\right. \\
& \left.+\frac{(\beta+s+(\alpha+\gamma) n)(\beta+s+k+(\alpha+\gamma) n)}{(\beta+s+2 k+(\alpha+\gamma) n)(\beta+s+k+(\alpha+\gamma) n)(\beta+s+(\alpha+\gamma) n) \Gamma_{k}((\beta+s+(\alpha+\gamma) n))}\right\} .
\end{aligned}
$$

Using (5), we have

$$
\begin{aligned}
S= & \sum_{n=0}^{\infty} \frac{\prod_{i=1}^{p} \Gamma_{k}\left(a_{i}+\alpha_{i} n\right)}{\prod_{j=1}^{q} \Gamma_{k}\left(b_{j}+\beta_{j} n\right) \Gamma_{k}(\beta+s+3 k+(\alpha+\gamma) n)} \frac{z^{n}}{n !} \\
& \times\left\{n^{2}(\alpha+\gamma)^{2}+2 n(\alpha+\gamma)(\beta+s+k)+(\beta+s)+(\beta+s+2 k)\right\}
\end{aligned}
$$

each summation in the right side of (16) can be expressed as follows:

$$
\begin{gathered}
\frac{d}{d z}\left\{z _ { p } \Psi _ { q + 1 } ^ { k } \left[\begin{array}{c}
\left.\left.\left(a_{i}, \alpha_{i}\right)_{1, p} \mid z\right]\right\} \\
\left(b_{j}, \beta_{j}\right)_{1, q},(\beta+s+3 k, \alpha+r) \mid z
\end{array}\right.\right. \\
=\sum_{n=0}^{\infty} \frac{\prod_{i=1}^{p} \Gamma_{k}\left(a_{i}+\alpha_{i} n\right)(n+1)}{\prod_{j=1}^{q} \Gamma_{k}\left(b_{j}+\beta_{j} n\right) \Gamma_{k}(\beta+s+3 k+(\alpha+\gamma) n)} \frac{z^{n}}{n !} \\
z_{p} \dot{\Psi}_{q+1}^{k}\left[\begin{array}{c}
\left.\left(b_{j}, \beta_{j}\right)_{1, q},(\beta+s+3 k, \alpha+r) \mid z\right] \\
\left.\left.+{ }_{p} \Psi_{q+1}^{k}\left[a_{i}, \alpha_{i}\right)_{1, p}, \beta_{j}\right)_{1, q},(\beta+s+3 k, \alpha+r) \mid z\right] \\
=\sum_{n=0}^{\infty} \frac{\left(a_{i}, \alpha_{i}\right)_{1, p}}{\prod_{j=1}^{q} \Gamma_{k}\left(b_{j}+\beta_{j} n\right) \Gamma_{k}(\beta+s+3 k+(\alpha+\gamma) n)}
\end{array}\right.
\end{gathered}
$$




$$
\begin{aligned}
z_{p} \dot{\Psi}_{q+1}^{k}\left[\left(b_{j}, \beta_{j}\right)_{1, q},(\beta+s+3 k, \alpha+r) \mid z\right] & \left.\left(a_{i}, \alpha_{i}\right)_{1, p}\right] \\
= & n \prod_{i=1}^{\infty} \frac{\Gamma_{k}\left(a_{i}+\alpha_{i} n\right)}{\prod_{j=1}^{q} \Gamma_{k}\left(b_{j}+\beta_{j} n\right) \Gamma_{k}(\beta+s+3 k+(\alpha+\gamma) n)} \frac{z^{n}}{n !} .
\end{aligned}
$$

Again

$$
\begin{gathered}
\frac{d^{2}}{d z^{2}}\left\{z_{p}^{2} \Psi_{q+1}^{k}\left[\left(b_{j}, \beta_{j}\right)_{1, q},(\beta+s+3 k, \alpha+r) \mid z\right]\right\} \\
=\sum_{n=0}^{\infty} \frac{\left.\prod_{i=1}^{p} \Gamma_{k}\left(a_{i}+\alpha_{i}\right)_{1, p}\right)(n+2)(n+1)}{\prod_{j=1}^{q} \Gamma_{k}\left(b_{j}+\beta_{j} n\right) \Gamma_{k}(\beta+s+3 k+(\alpha+\gamma) n)} \frac{z^{n}}{n !}
\end{gathered}
$$

Therefore

$$
\begin{aligned}
& z^{2}{ }_{p} \ddot{\Psi}_{q+1}^{k}\left[\begin{array}{c|c}
\left(a_{i}, \alpha_{i}\right)_{1, p} \\
\left(b_{j}, \beta_{j}\right)_{1, q},(\beta+s+3 k, \alpha+r)
\end{array} \mid z\right] \\
& +4 z_{p} \dot{\Psi}_{q+1}^{k}\left[\begin{array}{c|c}
\left(a_{i}, \alpha_{i}\right)_{1, p} \\
\left(b_{j}, \beta_{j}\right)_{1, q},(\beta+s+3 k, \alpha+r)
\end{array} \mid z\right] \\
& +2{ }_{p} \Psi_{q+1}^{k}\left[\begin{array}{c|c}
\left(a_{i}, \alpha_{i}\right)_{1, p} \\
\left(b_{j}, \beta_{j}\right)_{1, q},(\beta+s+3 k, \alpha+r)
\end{array} \mid z\right] \\
& =\sum_{n=0}^{\infty} \frac{\left(n^{2}+3 n+2\right) \prod_{i=1}^{p} \Gamma_{k}\left(a_{i}+\alpha_{i} n\right)}{\prod_{j=1}^{q} \Gamma_{k}\left(b_{j}+\beta_{j} n\right) \Gamma_{k}(\beta+s+3 k+(\alpha+\gamma) n)} \frac{z^{n}}{n !} .
\end{aligned}
$$

Using (17), above equation leads to

$$
\begin{gathered}
\sum_{n=0}^{\infty} \frac{n^{2} \prod_{i=1}^{p} \Gamma_{k}\left(a_{i}+\alpha_{i} n\right)}{\prod_{j=1}^{q} \Gamma_{k}\left(b_{j}+\beta_{j} n\right) \Gamma_{k}(\beta+s+3 k+(\alpha+\gamma) n)} \frac{z^{n}}{n !} \\
=z^{2} \ddot{\Psi}_{q+1}^{k}\left[\begin{array}{c}
\left.\left(b_{j}, \beta_{j}\right)_{1, q},(\beta+s+3 k, \alpha+r) \mid z\right] \\
+z_{p} \dot{\Psi}_{q+1}^{k}\left[\left(a_{j}, \beta_{j}\right)_{1, q},(\beta+s+3 k, \alpha+r) \mid z\right]
\end{array}\right.
\end{gathered}
$$


Substituting values of (17) and (18) in (16), yields

$$
\begin{aligned}
& S=z^{2}(\alpha+\gamma)^{2}{ }_{p} \ddot{\Psi}_{q+1}^{k}\left[\begin{array}{c}
\left(a_{i}, \alpha_{i}\right)_{1, p} \\
\left(b_{j}, \beta_{j}\right)_{1, q},(\beta+s+3 k, \alpha+r)
\end{array} \mid z\right] \\
& +z\left\{(\alpha+\gamma)^{2}+2(\alpha+\gamma)(\beta+s+k)\right\}_{p} \dot{\Psi}_{q+1}^{k}\left[\begin{array}{c}
\left(a_{i}, \alpha_{i}\right)_{1, p} \\
\left(b_{j}, \beta_{j}\right)_{1, q},(\beta+s+3 k, \alpha+r)
\end{array} \mid z\right] \\
& +(\beta+s)(\beta+s+2 k)_{p} \Psi_{q+1}^{k}\left[\begin{array}{c}
\left(a_{i}, \alpha_{i}\right)_{1, p} \\
\left(b_{j}, \beta_{j}\right)_{1, q},(\beta+s+3 k, \alpha+r)
\end{array} \mid z\right] .
\end{aligned}
$$

\section{Integral Representation}

In this section, authors established an intersting Integral representation of generalised $K$-Wright function ${ }_{p} \Psi_{q}^{k}(z)$.

Theorem 4.1. For $k \in \mathbb{R}^{+} ; z \in \mathbb{C} ; a_{i}, b_{j}, \beta+s \in \mathbb{C} ; \operatorname{Re}(\beta+s)>0$ and $\alpha+\gamma, \alpha_{i}, \beta_{j} \in \mathbb{R}\left(\alpha_{i}, \beta_{j} \neq 0 ; i=1,2, \ldots, p ; j=1,2, \ldots, q\right)$, then

$$
\begin{aligned}
& \int_{0}^{1} t^{\beta+s+k-1}{ }_{p} \Psi_{q+1}^{k}\left[\begin{array}{c}
\left(a_{i}, \alpha_{i}\right)_{1, p} \\
\left(b_{j}, \beta_{j}\right)_{1, q},(\beta+s, \alpha+r)
\end{array} \mid t^{\alpha+\gamma}\right] d t \\
& ={ }_{p} \Psi_{q+1}^{k}\left[\begin{array}{c|c}
\left(a_{i}, \alpha_{i}\right)_{1, p} & 1 \\
\left(b_{j}, \beta_{j}\right)_{1, q},(\beta+s+k, \alpha+r) & 1
\end{array}\right] \\
& -k_{p} \Psi_{q+1}^{k}\left[\begin{array}{c|c}
\left(a_{i}, \alpha_{i}\right)_{1, p} & \left(b_{j}, \beta_{j}\right)_{1, q},(\beta+s+2 k, \alpha+r)
\end{array} 1 .\right.
\end{aligned}
$$

Proof. Put $z=1$, in (14) and (15), we have

$$
\begin{gathered}
S={ }_{p} \Psi_{q+1}^{k}\left[\begin{array}{c}
\left(a_{i}, \alpha_{i}\right)_{1, p} \\
\left(b_{j}, \beta_{j}\right)_{1, q},(\beta+s+k, \alpha+\gamma)
\end{array} \mid 1\right] \\
-k_{p} \Psi_{q+1}^{k}\left[\begin{array}{c}
\left(a_{i}, \alpha_{i}\right)_{1, p} \\
\left(b_{j}, \beta_{j}\right)_{1, q},(\beta+s+2 k, \alpha+\gamma)
\end{array}\right] \\
=\sum_{n=0}^{\infty} \frac{\prod_{i=1}^{p} \Gamma_{k}\left(a_{i}+\alpha_{i} n\right)}{\prod_{j=1}^{q} \Gamma_{k}\left(b_{j}+\beta_{j} n\right)} \frac{1}{n !} \frac{1}{(\beta+s+k+(\alpha+\gamma) n) \Gamma_{k}(\beta+s+(\alpha+\gamma) n)} .
\end{gathered}
$$

Consider following integral

$$
A \equiv \int_{0}^{1} t^{\beta+s+k-1} \Psi_{q+1}^{k}\left[\begin{array}{c}
\left(a_{i}, \alpha_{i}\right)_{1, p} \\
\left(b_{j}, \beta_{j}\right)_{1, q},(\beta+s, \alpha+r)
\end{array} \mid t^{\alpha+\gamma}\right] d t .
$$


This can be written as

$$
A \equiv \sum_{n=0}^{\infty} \frac{\prod_{i=1}^{p} \Gamma_{k}\left(a_{i}+\alpha_{i} n\right)}{\prod_{j=1}^{q} \Gamma_{k}\left(b_{j}+\beta_{j} n\right)} \frac{1}{n !} \frac{1}{\Gamma_{k}(\beta+s+(\alpha+\gamma) n)} \int_{0}^{1} t^{(\alpha+\gamma) n+\beta+s+k-1} d t
$$

This immediately leads to

$$
A \equiv \sum_{n=0}^{\infty} \frac{\prod_{i=1}^{p} \Gamma_{k}\left(a_{i}+\alpha_{i} n\right)}{\prod_{j=1}^{q} \Gamma_{k}\left(b_{j}+\beta_{j} n\right)} \frac{1}{n !} \frac{1}{(\beta+s+k+(\alpha+\gamma) n) \Gamma_{k}(\beta+s+(\alpha+\gamma) n)}
$$

and from (20), we derive

$$
\begin{array}{r}
A={ }_{p} \Psi_{q+1}^{k}\left[\begin{array}{r}
\left(a_{i}, \alpha_{i}\right)_{1, p} \\
\left(b_{j}, \beta_{j}\right)_{1, q},(\beta+s+k, \alpha+\gamma)
\end{array}\right. \\
\quad-k_{p} \Psi_{q}^{k}\left[\begin{array}{r}
\left(b_{j}, \alpha_{i}\right)_{1, p} \\
\left(b_{j}\right)_{1, q},(\beta+s+2 k, \alpha+\gamma)
\end{array} \mid 1\right] .
\end{array}
$$

\section{Particular Case}

Taking $p=1, q=2 ; a_{1}=\gamma, \alpha_{1}=r, k=1 ; b_{1}=\beta, \beta_{1}=\alpha ; b_{2}=\gamma$, $\beta_{2}=0$, the results obtained by Shukla and Prajapati [4] also for suitable values of parameters the results obtained by Gupta and Debnath [3] are particular cases of results of this paper.

The function defined in equation (10) is an extension of generalized MittagLeffler function defined by Shukla and Prajapati (see [5], [6], [7]), and Shukla et al [8].

\section{References}

[1] R. Diaz, E. Pariguan, On hypergeometric functions and Pochhammer $k$ symbol, Divulgaciones Mathematicas, 15, No. 2 (2007), 179-192.

[2] K.S. Gehlot, J.C. Prajapati, On generalization of $K$-Wright function and its properties, Communicated for publication. 
[3] I.S. Gupta, L. Debnath, Some properties of the Mittag-Leffler functions, Integral Trans. Spec. Funct., 18, No. 5 (2007), 329-336.

[4] A.K. Shukla, J.C. Prajapati, On a recurrence relation of generalized Mittag-Leffler function, Surveys in Mathematics and its Applications, 4 (2009), 133-138.

[5] A.K. Shukla, J.C. Prajapati, A general class of polynomials associated with generalized Mittag-Lefflerfunction, Integral Transforms and Special Functions, 19, No. 1 (2008), 23-34.

[6] A.K. Shukla, J.C. Prajapati, Some remarks on generalized Mittag-Leffler function, Proyecciones Journal of Mathematics, 28, No. 1 (2009), 27-34.

[7] A.K. Shukla, J.C. Prajapati, On a generalized Mittag-Leffler type function and generated integral operator, Mathematical Sciences Research Journal, 12, No. 12 (2008), 283-290.

[8] A.K. Shukla, I.A. Salehbhai, J.C. Prajapati, On Laguerre Transform in two Variables, Integral Transforms and Special Functions, 20, No. 6 (2009), 459-470. 
